\title{
Radical palliative surgery: new limits to pursue
}

\author{
Mindy Young-Spint • Yigit S. Guner • \\ Frederick J. Meyers · Phillip Schneider • \\ Vijay P. Khatri
}

Accepted: 12 August 2009/Published online: 30 August 2009

(c) The Author(s) 2009. This article is published with open access at Springerlink.com

\begin{abstract}
This case report describes the radical subtotal palliative resection of a massive recurrent desmoid tumor encompassing the abdomen, pelvis, and groin in a child who was 13 years old at the time of initial resection. Given the extensive distribution of the tumor en bloc resection, which is the standard treatment of desmoid tumors, would have meant performing a hemipelvectomy and repair of a large abdominal wall defect, likely with skin grafts and mesh. The patient's personal goals however were to alleviate the pain and limited mobility that would allow her to re-attend high school and appear normal to her peers. Therefore, palliative surgery was pursued and currently the patient is 5 years out from her last surgery doing well. We believe that the option of surgical palliation in this case was warranted and should be an option for similar cases in the future.
\end{abstract}

Keywords Desmoid tumor - Palliative surgery ·

Pediatric $\cdot$ Palliation $\cdot$ Radical surgery

\section{P. Khatri $(\square)$}

University of California Davis School of Medicine, 2315 Stockton Blvd, Sacramento, CA 95817, USA

e-mail: vijay.khatri@ucdmc.ucdavis.edu

M. Young-Spint · Y. S. Guner · F. J. Meyers · P. Schneider University of California Davis Medical Center,

University of California Davis School of Medicine,

2315 Stockton Blvd, Sacramento, CA 95817, USA

e-mail: mindy.young-spint@ucdmc.ucdavis.edu

Y. S. Guner

e-mail: yigit.guner@ucdmc.ucdavis.edu

F. J. Meyers

e-mail: fredrick.meyers@ucdmc.ucdavis.edu

P. Schneider

e-mail: phillip.schneider@ucdmc.ucdavis.edu

\section{Introduction}

Patient-centered care defined by the Institute of Medicine is one quality indicator of care and includes informed consent and the support of patients' right to choose their level of treatment/palliation. Palliative care has been a widely accepted area of medicine that is incorporated into patients' daily lives who are dealing with terminal illnesses. It was initially incorporated only into end of life care and in earlier years was recognized as the equivalent to hospice, but current models stress the value of palliative care throughout illness. Palliative surgery is being examined within the context of advanced malignancy as well, and can be used with the intent of reducing symptoms to "maximize quality of life and minimize complications"[1].

Here, we are describing our experience of radical subtotal palliative resection of a massive recurrent desmoid tumor encompassing the abdomen, pelvis, and groin in a child who was 13 years old at the time of initial resection. We believe that, this case highlights the "balancing" act of: (1) pursuing "radical surgery" that can achieve cure, but leave the patient with significant functional deficits versus (2) performing palliative surgery to decrease the patient's symptoms from the tumor burden, still contending with the risks of surgery, but without completely treating their cancer. The radical treatment, given the extensive distribution of the tumor would have meant performing a hemipelvectomy and repair of a large abdominal wall defect, likely with skin grafts and mesh, after complete resection of her tumor. The patient's personal goals, however, were to alleviate the pain and limited mobility that would allow her to re-attend high school and appear normal to her peers, which a hemipelvectomy would not have achieved. The patient and her family would not consent to such a mutilating curative surgery, but opted to 
Fig. $1 \mathrm{CT}$ scan of the abdomen demonstrates the tumor displacing the small bowel (a) and more inferiorly the CT scan shows the tumor to extend over the pubic rami into the proximal thigh (b)
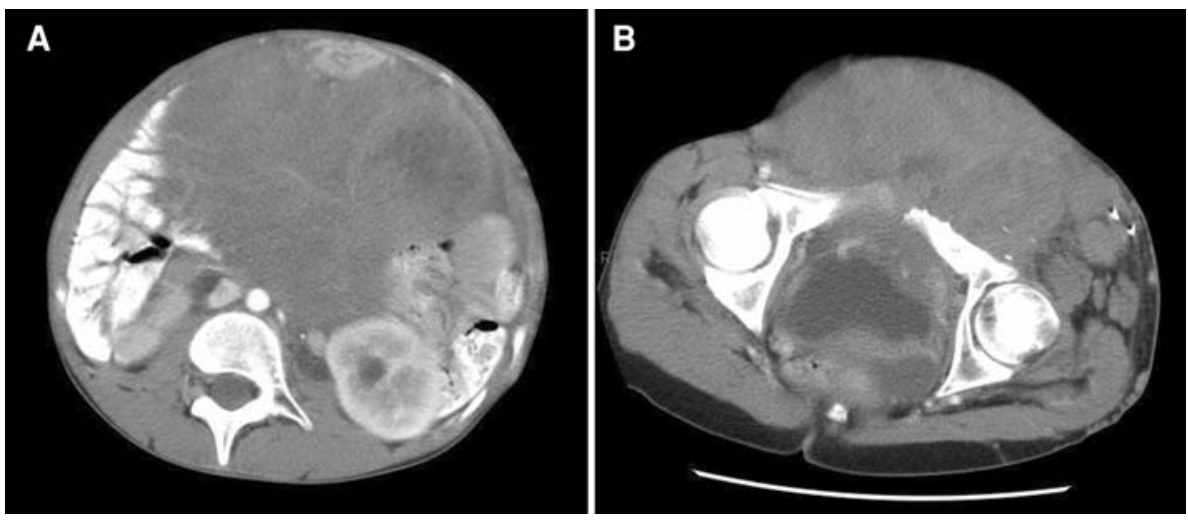

undergo a palliative operation that involved only partial resection of her tumor.

\section{Case description}

The patient is a 14-year-old girl who was referred to the UC Davis Surgical Oncology Clinic for the management of a massive desmoid tumor. At the time of presentation, she had developed almost near complete mechanical bowel obstruction and was failing to thrive. Many other centers had deemed her as a non-surgical candidate due to the extent of the disease.

Her history dates back to 1998 when she first developed a small left ankle mass. By biopsy this was confirmed to be an aggressive fibromatosis, namely, a desmoid tumor, which was resected at an outside institution. One year later, she developed a mass in the left groin, which biopsy again confirmed as a desmoid tumor. As this tumor was large and invading adjacent vascular structures she was treated with vinblastine and methotrexate, which resulted in initial stabilization of the disease.

However, with subsequent progression of the tumor in the left groin it was resected with en bloc removal of left femoral vein and the inguinal canal. The peritoneal contents were not violated during this resection and the defect in the deep pelvis was reconstructed with Marlex ${ }^{\circledR}$ mesh (Davol \& BARD Inc, Rhode Island).

Unfortunately, 4 years later the groin mass had recurred. It extended from the anteromedial compartment of the left lower extremity into the abdomen and pelvis as a massive desmoid tumor. After failing to respond to hormonal, antiinflammatory, and multi-agent chemotherapy she was referred to our center. The patient was symptomatic of this tumor to the point that she was bed bound, unable to tolerate oral intake, and had severe constant pain. Physical examination had revealed a slim young female who appeared in distress, trying to lie down whenever possible. The abdomen was distended with a large firm moderately tender mass, which extended to the level of the xiphoid process. It measured $21 \mathrm{~cm}$ in length in the midline, extending from the epigastrum to the pelvis and transversely, it extended from one flank to the other. In the left lower quadrant the tumor had eroded through the anterior abdominal wall. The left groin also had a firm, 14-cm mass with resulting difficulty in range of motion at the hip and subsequently decreased knee flexion.

CT scan demonstrated a large tumor occupying the abdomen, pelvic cavity and extending to the left proximal anteromedial thigh, displacing abdominal organs and causing bilateral hydronephrosis (Fig. 1). Due to the symptomatic nature of the tumor, the patient was unable to leave home and could only attend one outpatient clinic visit. Thus, the remaining counseling sessions were carried to her through her grandmother.

Given the extent of the tumor, to achieve complete resection the patient would have required a laparotomy to resect the abdominal and pelvic component with en bloc resection of the left lower quadrant anterior abdominal wall. In addition to address the groin and thigh component of the tumor with involvement of the neurovascular structures and the pelvic portion of the iliopsoas muscle (Fig. 1), an external hemipelvectomy was deemed necessary to achieve an R0 resection. Addition of the hemipelvectomy would have been a morbid surgical procedure with a negative impact on the patient's quality of life. The patient refused the external hemipelvectomy, as she was not willing to be wheelchair-bound and her goal was to not only attend school but also appear relatively normal to her peers. Since her symptoms were primarily from the burden of the abdomino-pelvic component of the tumor, palliative resection of this portion of the tumor was planned.

At the initial surgery, the tumor was approached through a midline incision with en bloc resection of the anterior abdominal wall. Intraoperatively, the bladder and left ureter were noted to be densely adherent to the posterior surface of the tumor. With the aid of cystoscopy, the bladder was identified and an en bloc resection of a $2 \mathrm{~cm}$ portion of the 


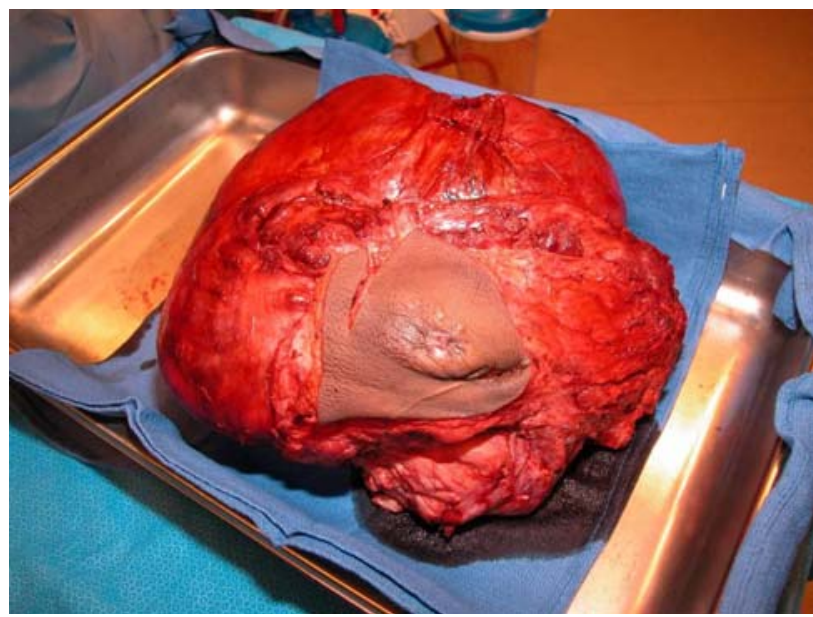

Fig. 2 Intraoperative view of the resected specimen

bladder wall and the distal part of the left ureter were completed. In addition, the adherent left fallopian tube, ovary, proximal sigmoid colon, and descending colon were removed with the specimen. Distally the dissection was taken to the left groin crease where the abdominal/pelvic tumor was contiguous with the groin mass. At this point some of the iliacus muscle was resected, and the last remnant of attachments from the pubic ramus was severed, amputating the tumor (measured $32 \times 26 \times 15 \mathrm{~cm}$ and weighed $6.1 \mathrm{~kg}$; Fig. 2). The bladder was then repaired and the left ureter was reimplanted. Resection of the anterior abdominal muscle and fascia, left a defect of $17 \times 17 \mathrm{~cm}$, with an overlying skin defect of $9 \times 9 \mathrm{~cm}$ (Fig. 3a).

Due to the duration of the initial surgical procedure, the decision was made to keep the patient on ventilatory support with the transected large bowel left in discontinuity secondary to bowel edema. Her abdomen was left open in order avoid intra-abdominal compartment syndrome and allow for adequate resuscitation. Temporary abdominal closure was performed with wound V.A.C. ${ }^{\circledR}$ (KCI Licensing Inc). She remained stable in Pediatric intensive care unit (PICU) for 3 days, when she was brought back for transverse colon to sigmoid colon anastomosis. The upper abdominal incision was closed leaving an open wound in the left lower quadrant dressed with a wound V.A.C ${ }^{\circledR}$.

Seven days after the initial operation the left lower quadrant abdominal wall defect was reconstructed with insetting of AlloDerm ${ }^{\circledR}$ (LifeCell Corp) (Fig. 3b). Thirtynine days after her discharge, she had excellent granulation tissue over the AlloDerm ${ }^{\circledR}$, and this area was covered with a split thickness skin graft $(14 \mathrm{~cm} \times 9 \mathrm{~cm})$. After recovery, the patient was able to ambulate, tolerate diet, and eventually return to school.

Seven months later, she had rapid growth of the groin desmoid tumor, which progressed after partial resection. Therefore, the groin and proximal thigh were treated with radiation therapy. Following this treatment, her tumor has demonstrated no growth during the 4 years of follow-up care.

\section{Discussion}

The World Health Organization defines palliative care as; "an approach that improves the quality of life of patients and their families facing the problem associated with lifethreatening illness, through the prevention and relief of suffering by means of early identification, and impeccable assessment and the treatment of pain and other problems, physical, psychosocial and spiritual."

Surgical treatment with wide margins is the primary treatment of desmoid tumors in children [2]. Survival from these tumors are favorable when local control is attainable [3]. The recurrence rate after surgery alone for the management of desmoids can be as high as $75 \%$ [4]. Radiation
Fig. 3 a Anterior abdominal wall defect after completion of the resection and $\mathbf{b}$ reconstruction of the lower abdominal wall defect with alloderm
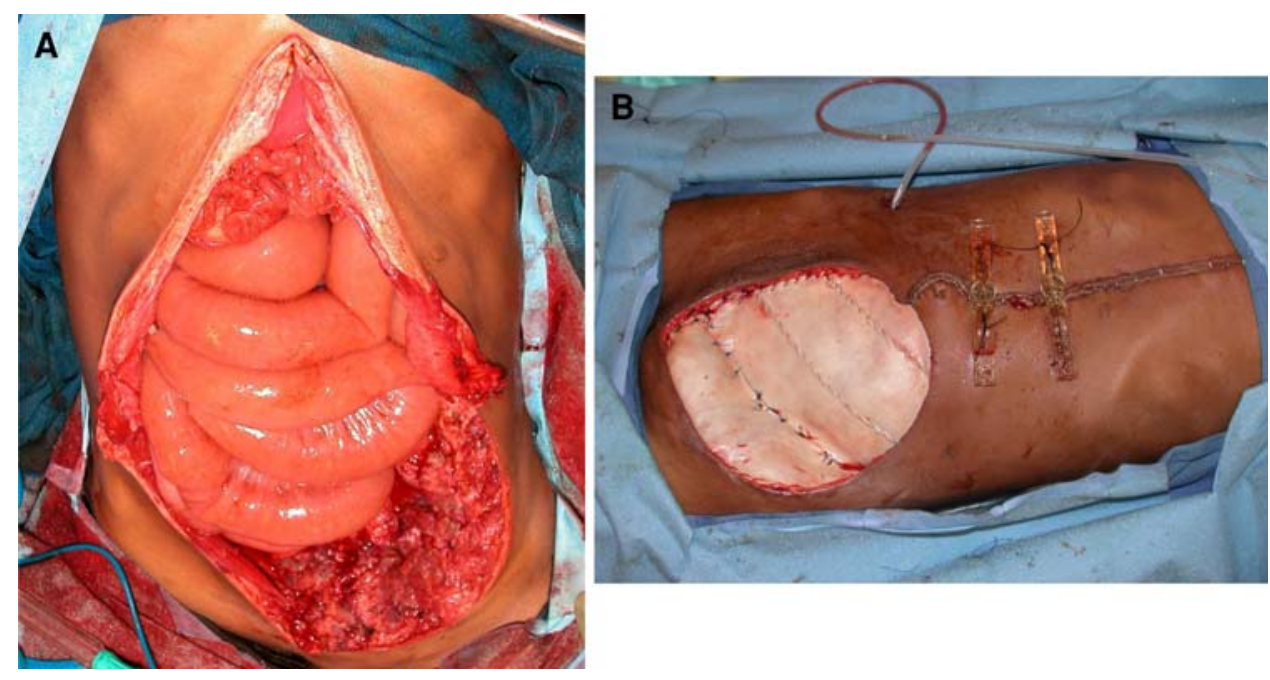
therapy can be employed, with a 10-year relapse rate of $25 \%$ in cases of positive resection margins. When radiation therapy has failed, NSAIDS and hormonal therapy is recommended [2, 3]. Also, combination cytotoxic chemotherapy with vinblastine and methotrexate has been reported with some success in children [2, 4] and adults [5]. Recurrent tumors can be life-threatening from their compressive symptoms, which commonly is the cause for patient's demise. In these situations complete resection should be considered, but when it is not anatomically feasible, current recommendations are to pursue medical management coupled with radiation [2, 4, 6, 7]. However, response rates are dismal and continued growth leads to an adverse impact on quality of life. Complete or subtotal resection should be entertained for palliation. Surgeons must consider each individual's need and appropriateness for surgery by a fine balancing act to maximize the quality of life and minimize complications.

The importance of this case herein lies with the successful long-term palliation we were able to provide by means of radical debulking and aggressive multi-disciplinary care.

The limited data regarding palliative surgery in the pediatric population expounds on the importance of early palliative discussions with parents, caregivers, and the patient, the barriers to care, the lack of evidence-based research at this time, and the need for further research $[8$, 9]. Dillon [10] discussed the increased need for surgical involvement in the treatment of children with cancer from vascular access issues and treatment of infection to the complications from chemotherapy, but there was no talk of palliative surgery.

Historically, the research in surgery and specifically palliative adult surgery has been measured by morbidity and mortality rates, but a few studies have looked at quality of life and symptom relief that palliative surgery is aimed towards, which will better assist clinicians and patients in deciding when palliation will benefit the patient [11-13]. Few studies at this time that have looked at quality of life and symptom relief in patients after palliative surgery have shown that $71-80 \%$ of patients have an improvement or resolution of symptoms achieved within 30 days and 54\% symptom free at 100 days [11-13].

Previous studies on palliative surgery have generally encompassed surgery for bowel obstruction, tumor debulking for symptoms of pain, and minor procedures $[1,2,11]$. For example, patients with gastrointestinal tumors also suffer symptoms from tumor growth including nausea/ vomiting and abdominal distention [2]. Surgical palliation in those cases focuses on resection to decrease the risk of bowel obstructions along with a decrease in symptoms. In a recent article, 35 patients with advanced, incurable gastrointestinal tumors were followed postoperatively for
6 months following palliative surgery and measurements in all areas for quality of life (physical, psychological, social and spiritual) showed that patients had decreased symptoms and an overall improvement in their quality of life [14].

This case required radical measures to be taken and exemplifies the spectrum that palliative surgery is capable of becoming to ensure a patient's quality of life and symptom management.

Recently, investigators described the importance of correctly classifying surgical procedures into the categories of palliative, non-curative, and curative [15]. The distinction between palliative surgery and non-curative surgery was statistically significant when measuring the median overall survival time. In palliative and curative surgeries the overall median survival time was 14.9 months [15], but non-curative operations were associated with decreased survival times of only 12.6 months [8]. Non-curative procedures were defined as surgical procedures with curative intent that, (1) during surgery metastatic or non-curable disease was identified, (2) the surgical procedure performed did not increase survival of the patient and (3) the surgery could not be labeled as palliative because excision of the mass was not in a symptomatic patient with quality of life issues [15]. This study raises a good point that further investigations in palliative surgery should only involve symptomatic patients or those patients who have such extreme disease noted that postponed surgery could cause complications like obstruction and bleeding. It is imperative that quality of life and symptom relief be addressed in these discussions with our patients.

Each patient is an individual and their care is "tailored" to their particular stage of illness, views on quality of life, and expectations of care. The relationship between the patient, the family, and the surgeon is paramount throughout cancer treatment and palliative care as there are many decisions to be made and different paths of treatment [15]. The communication between all parties must be clear and the patient's needs must be put first in all regards. Palliative surgery involves complex decision-making, but always for the best interest of the patient. In pediatric surgical oncology, the difficulty of decision-making is further confounded by the rarity and uniqueness of the condition.

In 2003, the American College of Surgeons Palliative task force formed a Surgeons Palliative Care Workgroup to analyze the current state of palliative care in the surgical field and render their recommendations for education, research, and clinical competencies. They found that further assessment is needed to develop targeted educational programs for surgeons in practice/training and establish funding sources and opportunities for research in surgical palliative care [16]. Also, further research must be done to consider the limits of palliative surgeries, but in the interim of such research it is 
possible to have similar outcomes in other patients who require such extensive and radical palliation.

These are challenging cases and raise the dilemma of deciding to take drastic measures with the risks of surgery, but without the goal of cure. However, palliative surgery is sometimes the best choice in a given situation based on the patient's goals. We believe that the surgeries in this case were justified based on the patient's goals and that the patient's autonomy was upheld. It is difficult at times when possible surgical or medical options are not available for treating particular diseases, but as physicians, we must always remember that in treating our patients: "To cure sometimes, to relieve often, and to comfort always" [17].

Open Access This article is distributed under the terms of the Creative Commons Attribution Noncommercial License which permits any noncommercial use, distribution, and reproduction in any medium, provided the original author(s) and source are credited.

\section{References}

1. Miner TJ, Jaques DP, Shriver CD (2002) A prospective evaluation of patients undergoing surgery for the palliation of an advanced malignancy. Ann Surg Oncol 9:696-703

2. Lackner H, Urban C, Benesch M et al (2004) Multimodal treatment of children with unresectable or recurrent desmoid tumors: an 11-year longitudinal observational study. J Pediatr Hematol Oncol 26:518-522

3. Mendenhall WM, Zlotecki RA, Morris CG, Hochwald SN, Scarborough MT (2005) Aggressive fibromatosis. Am J Clin Oncol 28:211-215

4. Skapek SX, Hawk BJ, Hoffer FA et al (1998) Combination chemotherapy using vinblastine and methotrexate for the treatment of progressive desmoid tumor in children. J Clin Oncol $16: 3021-3027$
5. Azzarelli A, Gronchi A, Bertulli R et al (2001) Low-dose chemotherapy with methotrexate and vinblastine for patients with advanced aggressive fibromatosis. Cancer 92:1259-1264

6. Mohos E, Kovacs T, Brittig F, Nagy A (2001) Desmoid tumors in three patients. Magy Seb 54:387-392

7. Ballo MT, Zagars GK, Pollack A, Pisters PW, Pollack RA (1999) Desmoid tumor: prognostic factors and outcome after surgery, radiation therapy, or combined surgery and radiation therapy. $\mathrm{J}$ Clin Oncol 17:158-167

8. Harris MB (2004) Palliative care in children with cancer: which child and when? J Natl Cancer Inst Monogr 2004:144-149

9. Mack JW, Wolfe J (2006) Early integration of pediatric palliative care: for some children, palliative care starts at diagnosis. Curr Opin Pediatr 18:10-14

10. Dillon PW (1999) Challenge of supportive surgical care in pediatric oncology. Semin Surg Oncol 16:193-199

11. Miner TJ, Brennan MF, Jaques DP (2004) A prospective, symptom related, outcomes analysis of 1022 palliative procedures for advanced cancer. Ann Surg 240:719-726 (discussion 26-27)

12. Yeh JJ, Singer S, Brennan MF, Jaques DP (2005) Effectiveness of palliative procedures for intra-abdominal sarcomas. Ann Surg Oncol 12:1084-1089

13. McCahill LE, Krouse RS, Chu DZ et al (2002) Decision making in palliative surgery. J Am Coll Surg 195:411-422 (discussion 22-23)

14. Podnos YD, Juarez G, Pameijer C, Uman G, Ferrell BR, Wagman LD (2007) Surgical palliation of advanced gastrointestinal tumors. J Palliat Med 10:871-876

15. McCahill LE, Smith DD, Borneman T et al (2003) A prospective evaluation of palliative outcomes for surgery of advanced malignancies. Ann Surg Oncol 10:654-663

16. Surgeon's Palliative Care Workgroup (2003) Robert Wood Johnson Foundation Office of Promoting Excellence in End-ofLife Care: executive summary of the report from the field. J Am Coll Surg 196:807-815

17. Surgeon's Palliative Care Workgroup (2003) Office of Promoting Excellence in End-of-Life Care: Surgeon's Palliative Care Workgroup report from the field. J Am Coll Surg 197:661-686 